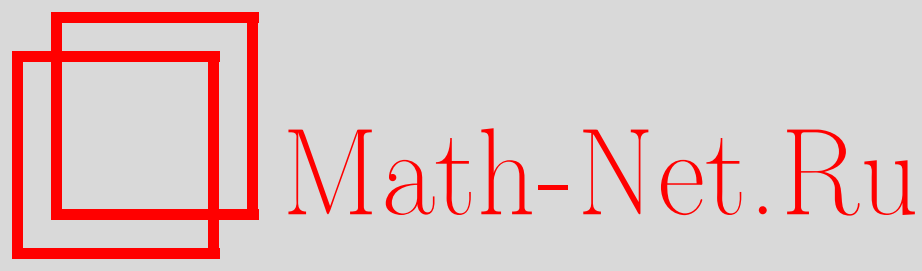

С. Л. Яковлев, Об асимптотическом поведении волновой функции трех частиц в континууме, ТM $\Phi, 2016$, том 186 , номер 1, 152-163

DOI: https://doi.org/10.4213/tmf8972

Использование Общероссийского математического портала Math-Net.Ru подразумевает, что вы прочитали и согласны с пользовательским соглашением http://www.mathnet.ru/rus/agreement

Параметры загрузки:

IP : 3.93.64.190

26 апреля 2023 г., $17: 39: 52$

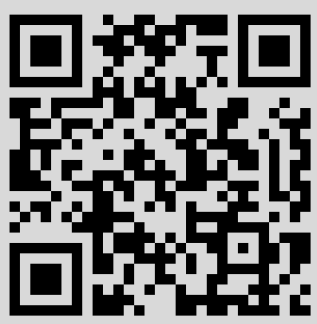




\title{
$\begin{array}{ll}\text { (C) } 2016 \text { г. } & \text { С. Л. Яковлев* }\end{array}$
}

\section{ОБ АСИМПТОТИЧЕСКОМ ПОВЕДЕНИИ ВОЛНОВОЙ ФУНКЦИИ ТРЕХ ЧАСТИЦ В КОНТИНУУМЕ}

\begin{abstract}
Исследуется волновая функция системы трех частиц в континууме. Сингулярности волновой функции в импульсном пространстве явно выделены с помощью уравнений Фаддеева. Асимптотическое поведение волновой функции в конфигурационном пространстве получено с помощью вычисления асимптотики преобразования Фурье волновой функции в импульсном пространстве. Основное внимание уделено конфигурациям, в которых две частицы находятся на относительно небольшом расстоянии друг от друга, в то время как третья частица значительно удалена от центра масс пары. Для таких конфигураций показано, что координатная асимптотика волновой функции помимо стандартных слагаемых содержит рассеянные волны нового типа. На основе полученных точных данных о координатной асимптотике волновой функции критически проанализирован мультипликативный анзац, используемый в ряде работ для описания системы трех частиц в континууме.
\end{abstract}

Ключевые слова: трехчастичное рассеяние, однократное и двукратное перерассеяние, асимптотика трехчастичной волновой функции в двухчастичном секторе.

DOI: $10.4213 / \operatorname{tmf} 8972$

Работа посвящена памяти академика С. П. Меркуръева в год семидесятилетия со дня рождения

\section{1. ВВЕДЕНИЕ}

Волновая функция для системы трех частиц, отвечающая рассеянию трех свободных частиц в начальном состоянии, исследовалась в работах ряда авторов [1]-[4]. Оказалось, что лишь использование уравнений Фаддеева [5], [6] позволило разобраться со сложным поведением волновой функции в конфигурационном пространстве путем выделения слагаемых, отвечающих процессам последовательных перерассеяний частиц. Было обнаружено, что помимо плоской волны и сферической

Работа поддержана СПбГУ (грант № 11.38.241.2015), РФФИ (грант № 14-0200326) и Программой поддержки ведущих научных школ (грант НШ-1292.2014.1).

* Санкт-Петербургский государственный университет, Санкт-Петербург, Россия. E-mail: s.yakovlev@spbu.ru 
волны в асимптотике волновой функции на больших расстояниях между частицами появляются "промежуточные" слагаемые, отвечающие однократным и двукратным перерассеяниям. Эти слагаемые убывают быстрее плоской волны, но медленнее сферической волны. При этом слагаемое, отвечающее двукратному перерассеянию, имеет разные асимптотические режимы в различных областях асимптотической части конфигурационного пространства. Смена асимптотического режима описывается с помощью специальной функции - интеграла Френеля. Различные аспекты такого характера асимптотики были обнаружены в работах [2]-[4]. Однако лишь в работе Меркурьева [1] был дан полный анализ ситуации и получен исчерпывающий результат по асимптотике всех характерных слагаемых, включая член двукратного перерассеяния, асимптотика которого впервые была получена в терминах интегралов Френеля именно в этой работе. Следует добавить, что впоследствии метод работы [1] был обобщен для изучения асимптотики волновой функции четырех частиц в работе [7].

В цитированных выше работах рассматривались конфигурации, в которых расстояния между всеми частицами являются большими. Такая область конфигурационного пространства называется трехчастичным сектором. Однако вся асимптотическая область не исчерпывается трехчастичным сектором, так как имеются конфигурации, в которых две частицы находятся на относительно небольшом расстоянии друг от друга, в то время как третья частица находится на большом расстоянии от центра масс выделенной пары. Такие конфигурации принято называть двухчастичным сектором. Обычно двухчастичный сектор связывают с реакциями образования связанных пар частиц при трехчастичных столкновениях [1]. Случай, когда все три частицы находятся в состоянии непрерывного спектра в двухчастичном секторе, является важным, однако он не исследован в деталях. Целью настоящей работы является построение асимптотики волновой функции трех частиц в двухчастичном секторе, что послужит дополнением к основной работе С. П. Меркурьева по трехчастичным асимптотикам [1]. Рассматривается случай частиц, взаимодействующих посредством короткодействующих потенциалов. Последнее обстоятельство, как и в [1], позволяет использовать уравнения Фаддеева для явного построения всех характерных слагаемых, отвечающих перерассеянию. Помимо собственной ценности полученная асимптотика имеет важное значение для обобщения на случай частиц с кулоновскими взаимодействиями. Дело в том, что в этом случае реализовать процедуру получения асимптотики с помощью изучения преобразований Фурье не представляется возможным [6], [8]. Как показано в работе [8], одним из конструктивных подходов в этом случае является использование прямых методов построения асимптотических решений уравнения Шредингера. При этом асимптотика волновой функции для короткодействующего случая используется как анзац, кулоновские поправки для которого и строятся прямыми методами.

Статья имеет следующую структуру. В разделе 2 дана постановка задачи и получена структура волновой функции трех частиц в импульсном представлении. В разделе 3 исследована асимптотика преобразований Фурье волновой функции в двухчастичном секторе. Раздел 4 посвящен использованию полученных асимптотических представлений для анализа области применимости так называемого мультипликативного анзаца. Статья завершается коротким заключением. 


\section{2. ПОСТАНОВКА ЗАДАЧИ}

Рассматривается система трех нерелятивистских бесспиновых частиц. После отделения движения центра масс конфигурационное пространство $\mathbb{Q}$ становится шестимерным пространством $\mathbb{R}^{6}$ с тремя системами координат Якоби $X=\left\{x_{\alpha}, y_{\alpha}\right\}$, $\alpha=1,2,3, x_{\alpha}, y_{\alpha} \in \mathbb{R}^{3}, X \in \mathbb{R}^{6}$. Разные системы координат связаны ортогональным преобразованием

$$
x_{\beta}=c_{\beta \alpha} x_{\alpha}+s_{\beta \alpha} y_{\alpha}, \quad y_{\beta}=-s_{\beta \alpha} x_{\alpha}+c_{\beta \alpha} y_{\alpha},
$$

где $\left[c_{\beta \alpha}\right]^{2}+\left[s_{\beta \alpha}\right]^{2}=1$ и эти коэффициенты зависят только от масс частиц [6], [9]. Гамильтониан относительного движения дается формулой

$$
H=H_{0}+V=H_{0}+\sum_{\beta=1}^{3} V_{\beta},
$$

где $H_{0}=-\Delta_{x_{\alpha}}-\Delta_{y_{\alpha}}$, а $V_{\beta}$ - операторы умножения на функции $v_{\beta}\left(x_{\beta}\right)$. Предполагается, что эти функции являются достаточно гладкими и быстроубывающими при $\left|x_{\beta}\right| \rightarrow \infty$. Точнее, предполагается, что преобразования Фурье функций $v_{\beta}\left(x_{\beta}\right)$ удовлетворяют условиям работы [5]. Такие потенциалы мы называем короткодействующими.

Переход в импульсное представление осуществляется преобразованием Фурье. Пространство $\mathbb{P}$ относительных импульсов, сопряженных координатам Якоби, состоит из пар $P=\left\{k_{\beta}, p_{\beta}\right\}$ с $k_{\beta}, p_{\beta} \in \mathbb{R}^{3}$ и $P \in \mathbb{R}^{6}$. При этом для любых индексов $\alpha=1,2,3$ выполняется равенство $P^{2}=k_{\alpha}^{2}+p_{\alpha}^{2}$. Относительные импульсы для разных индексов связаны ортогональными преобразованиями (1), в которых координаты следует заменить на соответствующие импульсы.

В настоящей работе изучаются волновые функции системы трех частиц $\Psi^{( \pm)}(P)$, которые отвечают свободному движению трех частиц в начальном $(+)$ или конечном (-) состоянии. Такие функции определяются следующим выражением [5]:

$$
\Psi^{( \pm)}\left(P^{\prime}\right)=\lim _{\epsilon \rightarrow+0}(\mp i \epsilon) R\left(P^{\prime 2} \pm i \epsilon\right) \Psi_{0}\left(P^{\prime}\right)
$$

в терминах резольвенты оператора $H$, определяемой формулой $R(z)=(H-z)^{-1}$, и собственной функции $\Psi_{0}\left(P^{\prime}\right)$ оператора $H_{0}$. Здесь и далее $i-$ мнимая единица. Будучи решением уравнения $H_{0} \Psi_{0}\left(P^{\prime}\right)=P^{2} \Psi_{0}\left(P^{\prime}\right)$, эта собственная функция в конфигурационном пространстве дается плоской волной:

$$
\Psi_{0}\left(X, P^{\prime}\right)=e^{i\left(P^{\prime}, X\right)},
$$

а в импульсном пространстве дельта-функцией:

$$
\Psi_{0}\left(P, P^{\prime}\right)=\delta\left(P-P^{\prime}\right) .
$$

Выражение $(P, X)$ обозначает скалярное произведение векторов в $\mathbb{R}^{6}$, а при использовании малых букв для векторов в $\mathbb{R}^{3}$ такое выражение обозначает скалярное произведение векторов в $\mathbb{R}^{3}$. 
Для нахождения $\Psi^{( \pm)}\left(P^{\prime}\right)$ будем следовать методике, предложенной в [1]. Предел в (3) легко вычисляется [5] с помощью ядра оператора

$$
T(z)=V-V R(z) V
$$

в импульсном пространстве

$$
\Psi^{( \pm)}\left(P, P^{\prime}\right)=\delta\left(P-P^{\prime}\right)-\frac{T\left(P, P^{\prime}, P^{\prime 2} \pm i 0\right)}{P^{2}-P^{\prime 2} \mp i 0} .
$$

Оператор $T(z)$, в свою очередь, выражается в терминах решений $M_{\alpha \beta}(z)$ уравнений Фаддеева

$$
M_{\alpha \beta}(z)=T_{\alpha}(z) \delta_{\alpha \beta}-T_{\alpha}(z) R_{0}(z) \sum_{\gamma \neq \alpha} M_{\gamma \beta}(z)
$$

суммой

$$
T(z)=\sum_{\alpha, \beta} M_{\alpha \beta}(z)
$$

Ядра операторов $T_{\alpha}(z)$ и $R_{0}(z)$, входящих в $(7)$, имеют вид

$$
\begin{aligned}
& T_{\alpha}\left(P, P^{\prime}, z\right)=t_{\alpha}\left(k_{\alpha}, k_{\alpha}^{\prime}, z-p_{\alpha}^{2}\right) \delta\left(p_{\alpha}-p_{\alpha}^{\prime}\right), \\
& R_{0}\left(P, P^{\prime}, z\right)=\left(P^{2}-z\right)^{-1} \delta\left(P-P^{\prime}\right)
\end{aligned}
$$

где $t_{\alpha}\left(k_{\alpha}, k_{\alpha}^{\prime}, z\right)$ - двухчастичная $T$-матрица, отвечающая потенциалу $v_{\alpha}$. Итерируя (7), придем к представлению

$$
M_{\alpha \beta}(z)=T_{\alpha}(z) \delta_{\alpha \beta}-T_{\alpha}(z) R_{0}(z) T_{\beta}(z) \bar{\delta}_{\alpha \beta}+W_{\alpha \beta}(z)
$$

где $\bar{\delta}_{\alpha \beta}=1-\delta_{\alpha \beta}$. Через $W_{\alpha \beta}(z)$ обозначены все более высокие итерации уравнений (7). В работе [5] показано, что слагаемое $W_{\alpha \beta}(z)$ может иметь лишь слабые сингулярности логарифмического типа, а в работе [1] показано, что такие сингулярности не влияют на вид старших членов асимптотики волновой функции. Поэтому с этим ядром в дальнейшем при вычислении асимптотики преобразований Фурье можно обращаться как с гладкой функцией. Ядро оставшегося второго слагаемого в (10) можно явно вычислить. Используя тот факт, что при $\alpha \neq \beta$ импульсы $p_{\alpha}$ и $p_{\beta}$ линейно независимы, для соответствующего интеграла легко получаем

$$
T_{\alpha}(z) R_{0}(z) T_{\beta}(z)\left(P, P^{\prime}\right)=\frac{t_{\alpha}\left(k_{\alpha}, k_{\alpha}\left(p_{\beta}^{\prime}, p_{\alpha}\right), z-p_{\alpha}^{2}\right) t_{\beta}\left(k_{\beta}\left(p_{\alpha}, p_{\beta}^{\prime}\right), k_{\beta}^{\prime}, z-p_{\beta}^{\prime 2}\right)}{\left|s_{\beta \alpha}\right|^{3}\left[k_{\beta}^{2}\left(p_{\alpha}, p_{\beta}^{\prime}\right)+p_{\beta}^{\prime 2}-z\right]} .
$$

Здесь импульсные переменные с аргументами даются выражениями

$$
k_{\alpha}\left(p_{\beta}^{\prime}, p_{\alpha}\right)=-\frac{1}{s_{\beta \alpha}} p_{\beta}^{\prime}+\frac{c_{\beta \alpha}}{s_{\beta \alpha}} p_{\alpha}, \quad k_{\beta}\left(p_{\alpha}, p_{\beta}^{\prime}\right)=-\frac{1}{s_{\alpha \beta}} p_{\alpha}+\frac{c_{\alpha \beta}}{s_{\alpha \beta}} p_{\beta}^{\prime} .
$$

С помощью полученных представлений выражение для $T\left(P, P^{\prime}, P^{\prime 2} \pm i 0\right)$ запишем в виде суммы трех групп слагаемых:

$$
T\left(P, P^{\prime}, P^{\prime 2} \pm i 0\right)=\sum_{\alpha} T_{\alpha}^{ \pm}\left(P, P^{\prime}\right)+\sum_{\alpha \neq \beta} T_{\alpha \beta}^{ \pm}\left(P, P^{\prime}\right)+W^{ \pm}\left(P, P^{\prime}\right) .
$$


Здесь слагаемые даются формулами

$$
\begin{aligned}
T_{\alpha}^{ \pm}\left(P, P^{\prime}\right) & =t_{\alpha}\left(k_{\alpha}, k_{\alpha}^{\prime}, k_{\alpha}^{\prime 2} \pm i 0\right) \delta\left(p_{\alpha}-p_{\alpha}^{\prime}\right), \\
T_{\alpha \beta}^{ \pm}\left(P, P^{\prime}\right) & =-\frac{t_{\alpha}\left(k_{\alpha}, k_{\alpha}\left(p_{\beta}^{\prime}, p_{\alpha}\right), P^{\prime 2}-p_{\alpha}^{2} \pm i 0\right) t_{\beta}\left(k_{\beta}\left(p_{\alpha}, p_{\beta}^{\prime}\right), k_{\beta}^{\prime}, k_{\beta}^{\prime 2} \pm i 0\right)}{\left|s_{\beta \alpha}\right|^{3}\left[k_{\beta}^{2}\left(p_{\alpha}, p_{\beta}^{\prime}\right)-k_{\beta}^{\prime 2} \mp i 0\right]} \\
W^{ \pm}\left(P, P^{\prime}\right) & =\sum_{\alpha, \beta} W_{\alpha \beta}\left(P, P^{\prime}, P^{\prime 2} \pm i 0\right) .
\end{aligned}
$$

Ясно, что структура (13) позволяет последовательно учесть вклады однократных столкновений с помощью $T_{\alpha}^{ \pm}$, двукратных столкновений с помощью $T_{\alpha \beta}^{ \pm}$и столкновений более высоких кратностей с помощью $W^{ \pm}$.

\section{3. ВОЛНОВАЯ ФУНКЦИЯ \\ В КОНФИГУРАЦИОННОМ ПРОСТРАНСТВЕ}

3.1. Строение волновой функции. Волновая функция в конфигурационном пространстве получается из $\Psi^{( \pm)}\left(P, P^{\prime}\right)$ с помощью обратного преобразования Фурье:

$$
\Psi^{( \pm)}\left(X, P^{\prime}\right)=\int d P e^{i(P, X)} \Psi^{( \pm)}\left(P, P^{\prime}\right)
$$

Выполняя интегрирование с использованием представлений (6) и (13), (14), получаем

$$
\Psi^{( \pm)}\left(X, P^{\prime}\right)=\Psi_{0}\left(X, P^{\prime}\right)+\sum_{\alpha} U_{\alpha}^{ \pm}\left(X, P^{\prime}\right)+\sum_{\alpha \neq \beta} U_{\alpha \beta}^{ \pm}\left(X, P^{\prime}\right)+U^{ \pm}\left(X, P^{\prime}\right)
$$

Слагаемые в (16) имеют следующий смысл. Первое слагаемое $\Psi_{0}\left(X, P^{\prime}\right)$ представляет плоскую волну и дается формулой (4). Однократные перерассеяния представлены слагаемыми $U_{\alpha}^{ \pm}\left(X, P^{\prime}\right)$, которые даются выражениями

$$
U_{\alpha}^{ \pm}\left(X, P^{\prime}\right)=u_{\alpha}^{ \pm}\left(x_{\alpha}, k_{\alpha}^{\prime}\right) e^{i\left(k_{\alpha}^{\prime}, x_{\alpha}\right)},
$$

в которых

$$
u_{\alpha}^{ \pm}\left(x_{\alpha}, k_{\alpha}^{\prime}\right)=-\int d k_{\alpha} e^{i\left(k_{\alpha}, x_{\alpha}\right)} \frac{t_{\alpha}\left(k_{\alpha}, k_{\alpha}^{\prime}, k_{\alpha}^{\prime 2} \pm i 0\right)}{k_{\alpha}^{2}-k_{\alpha}^{\prime 2} \mp i 0}
$$

является двухчастичной волновой функцией рассеяния за вычетом плоской волны и тем самым удовлетворяет уравнению

$$
\left[-\Delta_{x_{\alpha}}+v_{\alpha}\left(x_{\alpha}\right)-k_{\alpha}^{\prime 2}\right] u_{\alpha}^{ \pm}\left(x_{\alpha}, k_{\alpha}^{\prime}\right)=-v_{\alpha}\left(x_{\alpha}\right) e^{i\left(k_{\alpha}^{\prime}, x_{\alpha}\right)} .
$$

Двукратные перерассеяния представлены слагаемыми $U_{\alpha \beta}^{ \pm}\left(X, P^{\prime}\right)$, определяемыми интегралами:

$$
U_{\alpha \beta}^{ \pm}\left(X, P^{\prime}\right)=\int d P e^{i(P, X)} \frac{T_{\alpha \beta}^{ \pm}\left(P, P^{\prime}\right)}{P^{2}-P^{\prime 2} \mp i 0} .
$$


Наконец, перерассеяния всех более высоких кратностей представлены слагаемым $U^{ \pm}\left(X, P^{\prime}\right)$, которое дается аналогичным интегралом:

$$
U^{ \pm}\left(X, P^{\prime}\right)=-\int d P e^{i(P, X)} \frac{W^{ \pm}\left(P, P^{\prime}\right)}{P^{2}-P^{\prime 2} \mp i 0} .
$$

Для вычисления асимптотики интегралов типа (18) и (21) применяется следующее утверждение [6], доказательство которого легко получается с помощью методов из [10].

Лемма 1. Пусть $f(q, x), q, x \in \mathbb{R}^{n},-$ гладкая функиия переменных $q, x$ и медленно осциллирующая функиия переменной $x$ такая, что равномерно относительно $q$ выполняется неравенство

$$
\left|\nabla_{x} f(q, x)\right| \leqslant C(1+|x|)^{\nu}, \quad \nu<1 .
$$

Тогда асимптотика при $|x| \rightarrow \infty$ интеграла

$$
I(x, E)=\int d q e^{i(x, q)} \frac{f(q, x)}{q^{2}-E \mp i 0}
$$

дается выражением

$$
\begin{aligned}
I(x, E)=C_{n}(E)\left(\frac{1}{|x|}\right)^{(n-1) / 2} e^{\{ \pm i \sqrt{E}|x| \mp i \pi(n-3) / 4\}} \times \\
\times\left[f\left( \pm \frac{\sqrt{E} x}{|x|}, x\right)+O\left([\sqrt{E}|x|]^{-1+\nu}\right)\right],
\end{aligned}
$$

в котором $C_{n}(E)=(2 \pi)^{(n-1) / 2} \pi E^{(n-3) / 4}$.

С помощью этого утверждения легко показать, что величина $u^{ \pm}\left(x_{\alpha}, k_{\alpha}^{\prime}\right)$ из (18) при $\left|x_{\alpha}\right| \rightarrow \infty$ становится сферической волной в $\mathbb{R}^{3}$ и имеет порядок $O\left(\left|x_{\alpha}\right|^{-1}\right)$. Таким же образом при $|X| \rightarrow \infty$ величина $U^{ \pm}\left(X, P^{\prime}\right)$ из $(21)$ становится сферической волной в $\mathbb{R}^{6}$ и имеет порядок $O\left(|X|^{-5 / 2}\right)$. Более сложное поведение имеет величина $U_{\alpha \beta}^{ \pm}(X, P)$, отвечающая процессам двукратных последовательных столкновений частиц пар $\beta$ и $\alpha$. В работах [3] и [4] были получены утверждения о том, что величина $U_{\alpha \beta}^{ \pm}(X, P)$ при $\left|x_{\alpha}\right| \rightarrow \infty$ и $\left|y_{\alpha}\right| \rightarrow \infty$ имеет порядок $O\left(\left|x_{\alpha}\right|^{-1}\left|y_{\alpha}\right|^{-1}\right)$. Однако лишь в работе Меркурьева [1] было дано полное исследование асимптотического поведения $U_{\alpha \beta}^{ \pm}(X, P)$ при $\left|x_{\alpha}\right| \rightarrow \infty$ и $\left|y_{\alpha}\right| \rightarrow \infty$ и показано, что имеются две области в асимптотической части $\mathbb{Q}$, в одной из которых $U_{\alpha \beta}^{ \pm}(X, P) \sim O\left(\left|x_{\alpha}\right|^{-1}|X|^{-1}\right)$, а в другой $U_{\alpha \beta}^{ \pm}(X, P) \sim O\left(|X|^{-5 / 2}\right)$. Переходный режим при этом описывается интегралами Френеля. Мы не будем повторять здесь выкладки упомянутой работы. Заметим только, что все сделанные утверждения об асимптотике волновой функции $\Psi^{ \pm}\left(X, P^{\prime}\right)$ относятся к так называемому трехчастичному сектору, в котором для всех трех парных координат Якоби выполнено $\left|x_{\beta}\right| \rightarrow \infty, \beta=1,2,3$. В п. 3.2 мы рассмотрим случай, не охваченный приведенными здесь результатами, когда частица одной из пар в системе трех частиц могут находиться на относительно небольшом расстоянии, тогда как третья частица находится на большом расстоянии от данной пары. 
3.2. Асимптотика в двухчастичном секторе. Переходя к более точным утверждениям, мы будем предполагать, что относительные импульсы всех трех пар частиц отделены от нуля:

$$
\left|k_{\beta}^{\prime}\right| \geqslant \kappa>0, \quad \beta=1,2,3
$$

Двухчастичный сектор $\Omega_{\alpha}$ конфигурационного пространства $\mathbb{Q}$ тогда определяется неравенством [6]

$$
\left|x_{\alpha}\right| \leqslant a_{\alpha}\left(1+\left|y_{\alpha}\right|\right)^{\nu}, \quad \nu<1
$$

Будем считать, что точка $X$ находится в асимптотической части конфигурационного пространства, т. е. $|X| \gg 1$ и при этом $X \in \Omega_{\alpha}$. Последнее, в частности, означает, что $\left|y_{\alpha}\right| \gg 1$. Далее будем считать, что индекс $\alpha$ выделен и фиксирован и определяет двухчастичный сектор, в котором мы построим асимптотику волновой функции $\Psi^{( \pm)}\left(X, P^{\prime}\right)$. Выделим в (16) слагаемые, асимптотическое представление для которых необходимо получить в секторе $\Omega_{\alpha}$ :

$$
\begin{aligned}
\Psi^{( \pm)}\left(X, P^{\prime}\right)=\psi_{\alpha}^{( \pm)}\left(x_{\alpha}, k_{\alpha}^{\prime}\right) e^{i\left(p_{\alpha}^{\prime}, y_{\alpha}\right)}+\sum_{\beta \neq \alpha} U_{\beta}^{ \pm}\left(X, P^{\prime}\right)+ \\
+\sum_{\beta \neq \alpha} U_{\alpha \beta}^{ \pm}\left(X, P^{\prime}\right)+\sum_{\beta \neq \alpha} \sum_{\gamma \neq \beta} U_{\beta \gamma}^{ \pm}\left(X, P^{\prime}\right)+U^{ \pm}\left(X, P^{\prime}\right) .
\end{aligned}
$$

Здесь мы объединили первое и второе слагаемые из (16) и ввели обозначение

$$
\psi_{\alpha}^{( \pm)}\left(x_{\alpha}, k_{\alpha}^{\prime}\right)=e^{i\left(k_{\alpha}^{\prime}, x_{\alpha}\right)}+u_{\alpha}^{ \pm}\left(x_{\alpha}, k_{\alpha}^{\prime}\right)
$$

для двухчастичной волновой функции рассеяния на потенциале $v_{\alpha}\left(x_{\alpha}\right)$. В первом слагаемом в (24) лишь переменная $y_{\alpha}$ является большой, а плоская волна асимптотическому упрощению не подлежит, тем самым это слагаемое имеет порядок $O(1)$. Во второй группе слагаемых при $X \in \Omega_{\alpha}$ и $|X| \rightarrow \infty$ для $\beta \neq \alpha$ выполняется $\left|x_{\beta}\right| \sim|X|$ и, следовательно, $\left|x_{\beta}\right| \rightarrow \infty, \beta \neq \alpha$. По этой причине, применяя (22) при $n=3$ к функции $u_{\beta}^{ \pm}\left(x_{\beta}, k_{\beta}^{\prime}\right)$, получаем

$$
u_{\beta}^{ \pm}\left(x_{\beta}, k_{\beta}^{\prime}\right) \sim-C_{3}\left(k_{\beta}^{\prime 2}\right) t_{\beta}\left( \pm \frac{\left|k_{\beta}^{\prime}\right| x_{\beta}}{\left|x_{\beta}\right|}, k_{\beta}^{\prime}, k_{\beta}^{\prime 2} \pm i 0\right) \frac{e^{ \pm i\left|k_{\beta}^{\prime}\right|\left|x_{\beta}\right|}}{\left|x_{\beta}\right|},
$$

что дает для $U_{\beta}^{ \pm}\left(X, P^{\prime}\right)$

$$
U_{\beta}^{ \pm}\left(X, P^{\prime}\right) \sim-C_{3}\left(k_{\beta}^{\prime 2}\right) t_{\beta}\left( \pm \frac{\left|k_{\beta}^{\prime}\right| x_{\beta}}{\left|x_{\beta}\right|}, k_{\beta}^{\prime}, k_{\beta}^{\prime 2} \pm i 0\right) \frac{e^{i\left[ \pm\left|k_{\beta}^{\prime}\right|\left|x_{\beta}\right|+\left(p_{\beta}^{\prime}, y_{\beta}\right)\right]}}{\left|x_{\beta}\right|} .
$$

Тем самым показано, что $U_{\beta}^{ \pm}\left(X, P^{\prime}\right)$ имеет порядок $O\left(|X|^{-1}\right)$. Величину $Z_{\beta}^{ \pm}\left(X, P^{\prime}\right)$, которая определяется через выражение в показателе экспоненты формулой

$$
Z_{\beta}^{ \pm}\left(X, P^{\prime}\right)=\left|P^{\prime}\right|^{-1}\left[ \pm\left|k_{\beta}^{\prime}\right|\left|x_{\beta}\right|+\left(p_{\beta}^{\prime}, y_{\beta}\right)\right]
$$

принято называть однократным эйконалом [1], так как она, очевидно, удовлетворяет уравнению $\left|\nabla_{X} Z^{ \pm}\left(X, P^{\prime}\right)\right|^{2}=1$. 
Асимптотика последнего слагаемого в (24) при $|X| \rightarrow \infty$ также вычисляется с помощью (22) при $n=6$ и имеет вид

$$
U^{ \pm}\left(X, P^{\prime}\right) \sim-C_{6}\left(P^{\prime 2}\right) W^{ \pm}\left( \pm \frac{\left|P^{\prime}\right| X}{|X|}, P^{\prime}\right) \frac{e^{ \pm i\left|P^{\prime}\right||X| \mp i \pi 3 / 4}}{|X|^{5 / 2}} .
$$

Эти асимптотические представления совпадают с соответствующими представлениями работы [1], полученными для трехчастичного сектора конфигурационного пространства. Аналогично этому слагаемые $U_{\beta \gamma}^{ \pm}\left(X, P^{\prime}\right)$ при $\beta \neq \alpha$ в двухчастичном секторе $\Omega_{\alpha}$ пары $\alpha$ имеют поведение порядка $O\left(\left|x_{\beta}\right|^{-1}|X|^{-1}\right)$, полученное в [1]. Мы не будем повторять здесь соответствующие формулы из работы [1] из-за их громоздкости.

Таким образом, мы видим, что лишь слагаемые $U_{\alpha \beta}^{ \pm}\left(X, P^{\prime}\right)$ с $\beta \neq \alpha$ требуют специального исследования асимптотического поведения при $|X| \rightarrow \infty$ в двухчастичном секторе $\Omega_{\alpha}$. Для упрощения обозначений в дальнейшем будем рассматривать только случай функции $U_{\alpha \beta}^{+}\left(X, P^{\prime}\right)$, которая в явном виде дается интегралом

$$
\begin{aligned}
U_{\alpha \beta}^{+}\left(X, P^{\prime}\right)=\int & \frac{d k_{\alpha} d p_{\alpha}}{\left|s_{\beta \alpha}\right|^{3}} e^{i\left(k_{\alpha}, x_{\alpha}\right)} \frac{t_{\alpha}\left(k_{\alpha}, k_{\alpha}\left(p_{\beta}^{\prime}, p_{\alpha}\right), P^{\prime 2}-p_{\alpha}^{2}+i 0\right)}{k_{\alpha}^{2}-\left(P^{\prime 2}-p_{\alpha}^{\prime 2}\right)-i 0} \times \\
& \times e^{i\left(p_{\alpha}, y_{\alpha}\right)} \frac{t_{\beta}\left(k_{\beta}\left(p_{\alpha}, p_{\beta}^{\prime}\right), k_{\beta}^{\prime}, k_{\beta}^{\prime 2}+i 0\right)}{k_{\beta}^{2}\left(p_{\alpha}, p_{\beta}^{\prime}\right)-k_{\beta}^{\prime 2}-i 0} .
\end{aligned}
$$

Для интеграла по переменной $k_{\alpha}$ введем обозначение

$$
w_{\alpha}\left(x_{\alpha}, p_{\alpha}, P^{\prime}\right)=\int d k_{\alpha} e^{i\left(k_{\alpha}, x_{\alpha}\right)} \frac{t_{\alpha}\left(k_{\alpha}, k_{\alpha}\left(p_{\beta}^{\prime}, p_{\alpha}\right), P^{\prime 2}-p_{\alpha}^{2}-i 0\right)}{k_{\alpha}^{2}-\left(P^{\prime 2}-p_{\alpha}^{2}\right)-i 0} .
$$

Используя это обозначение и сделав замену переменной интегрирования $p_{\alpha}$ на $q_{\beta}=$ $k_{\beta}\left(p_{\alpha}, p_{\beta}^{\prime}\right)$, преобразуем выражение $(30)$ для $U_{\alpha \beta}^{+}\left(X, P^{\prime}\right)$ к следующему виду:

$$
\begin{aligned}
U_{\alpha \beta}^{+}\left(X, P^{\prime}\right)= & \left|\frac{s_{\alpha \beta}}{s_{\beta \alpha}}\right|^{3} e^{i\left(c_{\alpha \beta} p_{\beta}^{\prime}, y_{\alpha}\right)} \times \\
& \times \int d q_{\beta} e^{i\left(q_{\beta},-s_{\alpha \beta} y_{\alpha}\right)} \frac{t_{\beta}\left(q_{\beta}, k_{\beta}^{\prime}, k_{\beta}^{\prime 2}+i 0\right) w_{\alpha}\left(x_{\alpha}, p_{\alpha}\left(q_{\beta}, p_{\beta}^{\prime}\right), P^{\prime}\right)}{q_{\beta}^{2}-k_{\beta}^{\prime 2}-i 0} .
\end{aligned}
$$

Далее мы воспользуемся тем, что в секторе $\Omega_{\alpha}$ для переменной $x_{\alpha}$ выполняется неравенство (23), а тогда, как непосредственно видно из определения (31), функция $w_{\alpha}\left(x_{\alpha}, p_{\alpha}\left(q_{\beta}, p_{\beta}^{\prime}\right), P^{\prime}\right)$ является медленно осциллирующей функцией переменной интегрирования; точнее, при $\left|y_{\alpha}\right| \rightarrow \infty$ выполняется асимптотическая оценка

$$
\nabla_{q_{\beta}} w_{\alpha}\left(x_{\alpha}, p_{\alpha}\left(q_{\beta}, p_{\beta}^{\prime}\right), P^{\prime}\right) \sim O\left(\left|y_{\alpha}\right|^{\nu}\right), \quad \nu<1
$$

Это свойство позволяет воспользоваться асимптотикой (22) из леммы 1 для вычисления асимптотики при $\left|y_{\alpha}\right| \rightarrow \infty$ интеграла в $(32)$. Таким образом, для $U_{\alpha \beta}^{+}\left(X, P^{\prime}\right)$ получаем

$$
\begin{aligned}
U_{\alpha \beta}^{+}\left(X, P^{\prime}\right) \sim & C_{3}\left(k_{\beta}^{\prime 2}\right) \frac{\left|s_{\alpha \beta}\right|^{2}}{\left|s_{\beta \alpha}\right|^{3}} \frac{e^{i\left[\left|s_{\alpha \beta} k_{\beta}^{\prime}\right|\left|y_{\alpha}\right|+\left(c_{\alpha \beta} p_{\beta}^{\prime}, y_{\alpha}\right)\right]}}{\left|y_{\alpha}\right|} \times \\
& \times t_{\beta}\left(-\left|k_{\beta}^{\prime}\right| \widehat{y}_{\alpha}, k_{\beta}^{\prime}, k_{\beta}^{\prime 2}+i 0\right) w_{\alpha}\left(x_{\alpha}, p_{\alpha}\left(-\left|k_{\beta}^{\prime}\right| \widehat{y}_{\alpha}, p_{\beta}^{\prime}\right), P^{\prime}\right),
\end{aligned}
$$


где для единичного вектора $y_{\alpha} /\left|y_{\alpha}\right|$ введено обозначение $\widehat{y}_{\alpha}$. Заметим, что при переходе точки $X$ из двухчастичного сектора $\Omega_{\alpha}$ в трехчастичный сектор, в котором величина $\left|x_{\alpha}\right|$ становится одного порядка с $\left|y_{\alpha}\right|$, асимптотика $U_{\alpha \beta}^{+}\left(X, P^{\prime}\right)$ не может быть получена из (34) простым взятием асимптотики $w_{\alpha}\left(x_{\alpha}, p_{\alpha}\left(-\left|k_{\beta}^{\prime}\right| \widehat{y}_{\alpha}, p_{\beta}^{\prime}\right), P^{\prime}\right)$ при $\left|x_{\alpha}\right| \rightarrow \infty$. Это связано с тем, что для того чтобы получить (34) из (32), необходимо выполнение условия (33), которое выполняется только в секторе $\Omega_{\alpha}$ и нарушается при выходе в трехчастичный сектор. Для получения асимптотического поведения волновой функции в трехчастичном секторе нужно использовать методы работы [1].

Итак, мы получили асимптотику всех слагаемых в представлении волновой функции $\Psi^{(+)}\left(X, P^{\prime}\right)$ в двухчастичном секторе. Эти слагаемые имеют порядки $O(1)$, $O\left(|X|^{-1}\right), O\left(|X|^{-2}\right)$ и $O\left(|X|^{-5 / 2}\right)$. Важно отметить, что помимо стандартных членов порядка $O\left(|X|^{-1}\right)$ типа сферической волны (27) мы обнаружили нестандартную волну (34), имеющую порядок $O\left(|X|^{-1}\right)$ и не сводящуюся к простой сферической волне. Отличительной чертой этой волны является то, что она формируется процессами двукратных перерассеяний, в отличие от стандартных сферических волн (27), которые формируются процессами однократных столкновений.

\section{4. МУЛЬТИПЛИКАТИВНЫЙ АНЗАЦ}

Мультипликативный анзац был впервые введен в работе [11] для описания старшего члена волновой функции трех заряженных частиц в трехчастичном секторе в окрестности двухчастичных направлений рассеяния вперед. Впоследствии этот анзац был использован и в двухчастичном секторе [12], [13]. Причем в последней работе и ряде последующих работ этот анзац был модифицирован для улучшения его точности как решения уравнения Шредингера в двухчастичном секторе ${ }^{1)}$. Здесь мы сравним детали этого анзаца с представлением (24), являющимся точным представлением для волновой функции как следствие уравнений Фаддеева. Мультипликативным анзацем называют выражение

$$
A\left(X, P^{\prime}\right)=e^{i\left(X, P^{\prime}\right)} F_{1}\left(x_{1}, k_{1}^{\prime}\right) F_{2}\left(x_{2}, k_{2}^{\prime}\right) F_{3}\left(x_{3}, k_{3}^{\prime}\right) .
$$

Действуя на $A\left(X, P^{\prime}\right)$ оператором $H_{0}+V-P^{\prime 2}$, приходим к выражению

$$
\begin{aligned}
e^{-i\left(P^{\prime}, X\right)}\left(H_{0}+V-P^{\prime 2}\right) A\left(X, P^{\prime}\right)= & \sum_{\{\alpha, \beta, \gamma\}}\left[-\Delta_{x_{\alpha}} F_{\alpha}-2 i\left(k_{\alpha}, \nabla_{x_{\alpha}} F_{\alpha}\right)+V_{\alpha} F_{\alpha}\right] F_{\beta} F_{\gamma}- \\
& -\sum_{\{\alpha, \beta, \gamma\}}\left(\nabla_{x_{\alpha}} F_{\alpha}, c_{\beta \alpha} \nabla_{x_{\beta}} F_{\beta}\right) F_{\gamma}
\end{aligned}
$$

в котором суммирование ведется по циклическим перестановкам основной перестановки $\{1,2,3\}$. Первая сумма в правой части (36) исчезает при условии

$$
-\Delta_{x_{\alpha}} F_{\alpha}-2 i\left(k_{\alpha}^{\prime}, \nabla_{x_{\alpha}} F_{\alpha}\right)+V_{\alpha} F_{\alpha}=0
$$

1) Не претендуя на полноту литературных ссылок, мы даем здесь ссылки только на основные работы по данной теме. 
Решение этого уравнения дается формулой

$$
F_{\alpha}\left(x_{\alpha}, k_{\alpha}^{\prime}\right)=e^{-i\left(k_{\alpha}^{\prime}, x_{\alpha}\right)} \psi_{\alpha}^{+}\left(x_{\alpha}, k_{\alpha}^{\prime}\right)
$$

в терминах двухчастичной волновой функции $\psi_{\alpha}^{+}\left(x_{\alpha}, k_{\alpha}^{\prime}\right)$, удовлетворяющей уравнению

$$
\left[-\Delta_{x_{\alpha}}+v_{\alpha}\left(x_{\alpha}\right)-k_{\alpha}^{\prime 2}\right] \psi_{\alpha}^{+}\left(x_{\alpha}, k_{\alpha}^{\prime}\right)=0 .
$$

В результате из (36) получаем

$$
\left(H_{0}+V-P^{\prime 2}\right) A\left(X, P^{\prime}\right)=-e^{-i\left(P^{\prime}, X\right)} \sum_{\{\alpha, \beta, \gamma\}}\left(\nabla_{x_{\alpha}} F_{\alpha}, c_{\beta \alpha} \nabla_{x_{\beta}} F_{\beta}\right) F_{\gamma} .
$$

Заметим, что полученные представления фактически не зависят от типа входящих в уравнение Шредингера потенциалов и, в частности, годны также для случая кулоновских потенциалов $v_{\alpha}\left(x_{\alpha}\right)=n_{\alpha} /\left|x_{\alpha}\right|$.

Правая часть уравнения (40) является убывающей функцией при $|X| \rightarrow \infty$, и, следовательно, $A\left(X, P^{\prime}\right)$ при этом становится асимптотическим решением уравнения Шредингера

$$
\left(H_{0}+\sum_{\alpha=1}^{3} V_{\alpha}-P^{\prime 2}\right) \Psi^{+}\left(X, P^{\prime}\right)=0 .
$$

В трехчастичном секторе конфигурационного пространства $\mathbb{Q}$, где выполняется условие $\left|x_{\alpha}\right| \sim|X| \gg 1$ для всех $\alpha=1,2,3$, для правой части (40) имеем

$$
-e^{-i\left(P^{\prime}, X\right)} \sum_{\{\alpha, \beta, \gamma\}}\left(\nabla_{x_{\alpha}} F_{\alpha}, c_{\beta \alpha} \nabla_{x_{\beta}} F_{\beta}\right) F_{\gamma} \sim O\left(|X|^{-2}\right) .
$$

Последнее является следствием того, что из определения (38) и представления (26) при $\left|x_{\beta}\right| \rightarrow \infty$ следует элементарная оценка

$$
\nabla_{x_{\beta}} F_{\beta}\left(x_{\beta}, k_{\beta}^{\prime}\right) \sim O\left(\left|x_{\beta}\right|^{-1}\right) .
$$

Из данных оценок непосредственно следует, что анзац $A\left(X, P^{\prime}\right)$ содержит избыточную информацию, так как ввиду (42) все члены порядка $O\left(|X|^{-2}\right)$ и более высоких порядков малости следует удалить из представления (35), что ведет к равенству

$$
A\left(X, P^{\prime}\right)=e^{i\left(P^{\prime}, X\right)}\left[1+\sum_{\alpha} e^{-i\left(k_{\alpha}^{\prime}, x_{\alpha}\right)} u_{\alpha}^{+}\left(x_{\alpha}, k_{\alpha}^{\prime}\right)\right] .
$$

Правая часть данного равенства совпадает с двумя первыми членами представления (16) и тем самым содержит лишь члены, отвечающие свободному движению и процессам однократных столкновений. Улучшение качества аппроксимации волновой функции $\Psi^{+}\left(X, P^{\prime}\right)$ с помощью $A\left(X, P^{\prime}\right)$ из (44) может быть достигнуто только учетом следующих по порядку слагаемых из (16). Этих слагаемых с очевидностью нет в исходном выражении (35). Таким образом, мы установили, что анзац (35) в его оригинальной форме позволяет получить решение уравнения Шредингера с точностью не лучше $O\left(|X|^{-2}\right)$. Улучшение точности решения возможно лишь добавлением поправочных к (44) слагаемых из (16). Тем самым эффективность использования анзаца (35) для построения асимптотического решения уравнения Шредингера (41) в трехчастичном секторе ограничивается лишь случаем самых старших членов в асимптотическом разложении. 
Рассмотрим эффективность анзаца в двухчастичных секторах. В этом случае оценка (42) ухудшается. Рассмотрим, например, двухчастичный сектор $\Omega_{1}$. В этом секторе для $\nabla_{x_{1}} F_{1}$ теперь выполняется лишь оценка $\nabla_{x_{1}} F_{1} \sim O(1)$. В то же время при $\beta \neq 1$ по-прежнему $\left|x_{\beta}\right| \sim|X|$ и выполняется оценка $\nabla_{x_{\beta}} F_{\beta} \sim O\left(\left|x_{\beta}\right|^{-1}\right)$. Таким образом, в $\Omega_{1}$ выполняется соотношение

$$
-e^{-i\left(P^{\prime}, X\right)} \sum_{\{\alpha, \beta, \gamma\}}\left(\nabla_{x_{\alpha}} F_{\alpha}, c_{\beta \alpha} \nabla_{x_{\beta}} F_{\beta}\right) F_{\gamma} \sim O\left(|X|^{-1}\right)
$$

Вновь данная оценка показывает избыточность (35). В данном случае в выражении для $A\left(X, P^{\prime}\right)$ следует оставить только члены порядка $O(1)$ :

$$
A\left(X, P^{\prime}\right)=e^{i\left(P^{\prime}, X\right)}\left[1+e^{-i\left(k_{1}^{\prime}, x_{1}\right)} u_{1}^{+}\left(x_{1}, k_{1}^{\prime}\right)\right] .
$$

Вновь улучшение качества аппроксимации волновой функции $\Psi^{+}\left(X, P^{\prime}\right)$ с помощью $A\left(X, P^{\prime}\right)$ из (46) может быть достигнуто только добавлением следующих по порядку групп слагаемых из (16) или (24):

$$
A\left(X, P^{\prime}\right)=e^{i\left(P^{\prime}, X\right)}\left[1+e^{-i\left(k_{1}^{\prime}, x_{1}\right)} u_{1}^{+}\left(x_{1}, k_{1}^{\prime}\right)\right]+\sum_{\beta \neq 1} U_{\beta}^{+}\left(X, P^{\prime}\right)+\sum_{\beta \neq 1} U_{1 \beta}^{+}\left(X, P^{\prime}\right) .
$$

Данное представление с очевидностью позволяет получить решение уравнения Шредингера (41) с точностью $O\left(|X|^{-2}\right)$. Дальнейшее улучшение точности решения требует добавления следующих по порядку членов из (24). Таким образом, как и выше, мы установили, что анзац (35) в его оригинальной форме позволяет получить решение уравнения Шредингера с точностью не лучше $O\left(|X|^{-1}\right)$. Улучшение точности решения возможно лишь добавлением поправочных к (44) слагаемых из (24). Очевидно, что получаемое таким образом представление для асимптотического решения уже не имеет ничего общего с исходным анзацем (35).

\section{5. ЗАКЛЮЧЕНИЕ}

В работе построено асимптотическое представление для волновой функции трех частиц в двухчастичных секторах асимптотической части конфигурационного пространства. Показано, что в асимптотике волновой функции трех частиц к слагаемым, описывающим процессы свободного движения и однократных перерассеяний, добавляется нестандартное слагаемое, отвечающее процессам двукратных перерассеяний, однако имеющее тот же порядок $O\left(|X|^{-1}\right)$, что и члены однократных перерассеяний. На основе полученных представлений для волновой функции проанализировано качество мультипликативного анзаца. Показано, что фактически этот анзац пригоден для аппроксимации лишь самых старших членов асимптотического представления волновой функции, как это и было предложено в оригинальной работе [11]. Полученные в настоящей работе асимптотические представления для волновой функции трех частиц, взаимодействующих посредством короткодействующих потенциалов, будут весьма полезны для построения эффективного асимптотического представления для волновой функции трех кулоновских частиц. 


\section{Список литературы}

[1] С. П. Меркурьев, ТМФ, 8:2 (1971), 235-250.

[2] E. Gerjuoy, J. Phys. B, 3:8 (1970), L92-L97; Phil. Trans. Royal Soc. London. Ser. A, 270:1203 (1971), 197-287.

[3] J. Nuttal, J. Math. Phys., 12:9 (1971), 1896-1899.

[4] R. G. Newton, Ann. Phys., 74:2 (1972), 324-351.

[5] Л. Д. Фаддеев, Тр. МИАН СССР, 69 (1963), 1-122.

[6] С. П. Меркурьев, Л. Д. Фаддеев, Квантовая теория рассеяния для систем нескольких частии, Наука, М., 1985.

[7] С. Л. Яковлев, ТМФ, 82:2 (1990), 224-241.

[8] С. П. Меркурьев, ТМФ, 32:2 (1977), 187-207.

[9] С. Л. Яковлев, ТМФ, 181:1 (2014), 218-240.

[10] М. В. Федорюк, Метод перевала, Наука, М., 1977.

[11] С. П. Меркурьев, ТМФ, 38:2 (1979), 201-218.

[12] H. Klar, Z. Phys. D, 16:4 (1990), 231-236.

[13] E. O. Alt, A. M. Mukhamedzhanov, Phys. Rev. A, 47:3 (1993), 2004-2022. 